\title{
Does animal model on ventilator-associated pneumonia reflect physiopathology of sepsis mechanisms in humans?
}

\author{
Laura Pulido $^{1}$, Diego Burgos ${ }^{1}$, Joaquín García Morato ${ }^{2}$, Carlos M. Luna ${ }^{1}$ \\ ${ }^{1}$ Department of Pulmonary Medicine, ${ }^{2}$ Thoracic Surgery Division, Department of Surgery, Experimental Surgery University Center, Hospital de \\ Clínicas José de San Martín, Universidad de Buenos Aires, Buenos Aires, Argentina \\ Contributions: (I) Conception and design: All authors; (II) Administrative support: None; (III) Provision of study materials or patients: All authors; (IV) \\ Collection and assembly of data: All authors; (V) Data analysis and interpretation: None; (VI) Manuscript writing: All authors; (VII) Final approval of \\ manuscript: All authors. \\ Correspondence to: Carlos M. Luna, MD, PhD. Arenales 2557, piso 1, dto A, Ciudad Autónoma de Buenos Aires, Capital Federal, CP 1425, Argentina. \\ Email: dr.cm.luna@gmail.ar.
}

\begin{abstract}
Ventilator-associated pneumonia (VAP) is the leading cause of death in critically ill patients in intensive care units. In the last 20 years, different animal models have been a valuable tool for the study of pathophysiology and phenotypic characteristics of different lung infections observed in humans, becoming an essential link between "in vitro" testing and clinical studies. Different animal models have been used to study the mechanism of a deregulated inflammatory response and host tissue damage of sepsis in VAP, as well as different infection parameters such as clinical, physiological, microbiological and pathological facts in several large and small mammals. In addition, the dosage of inflammatory modulators and their consequences in local and systemic inflammation, or even the administration of antibiotics, have been evaluated with very interesting results. Although some bronchial inoculation ways do not resemble the common pathophysiologic mechanisms, the experimental model of VAP induced by the inoculation of high concentrations of pathogens in mechanically ventilated animals is useful for studying the local and systemic responses of sepsis in VAP and it reproduces biological mechanisms such as acute lung injury, distress response, cardiac events and immune modulation comparable with clinical studies
\end{abstract}

Keywords: Animal model; ventilator-associated pneumonia (VAP); inflammatory markers; antimicrobial therapy; sepsis mechanisms

Submitted Nov 18, 2017. Accepted for publication Nov 21, 2017.

doi: $10.21037 /$ atm.2017.11.35

View this article at: http://dx.doi.org/10.21037/atm.2017.11.35

\section{Introduction}

Ventilator-associated pneumonia (VAP) is considered pneumonia in patients intubated and receiving invasive mechanical ventilation (MV) for more than 48 hours. The diagnosis of VAP is based on the presence of a new radiographic infiltrate plus 2 out of 3 of the following conditions: fever or hypothermia; leukocytosis or leucopenia and/or purulent tracheobronchial secretions. In the ICU, the risk of mortality appears to be 2- to 10-fold higher in patients with VAP and may be as high as $70 \%(1,2)$.

According to the third international consensus definitions for sepsis and septic shock (Sepsis-3), sepsis should be defined as life-threatening organ dysfunction caused by a dysregulated host response to infection (3). Sepsis is the primary cause of death from infection; its recognition mandates urgent attention. Pathogen factors and host factors shape this syndrome: sex, age, comorbidities, environment, etc., with characteristics that evolve over time. Animal models may be useful to overcome some of the obstacles, due to different factors, to understand their pathophysiology and to develop new approaches for the management of this syndrome. For this type of models in vivo, sepsis is reached when animals have an infection and 
demonstrate 'toxicity' (fever, anorexia, weight loss, or other signs of illness) (4). Such important issues compose the complexity of this multifactorial disease (5).

In the last two decades, animal models and in vitro analyses, in combination with human population studies, were essential to understand the complex relationship between the microbiology and on the other hand, the inflammatory response to infection in VAP $(6,7)$. Literally hundreds of different animal models of sepsis have been used by scientists (8).

The objectives of this review are to present the major animal models used in translational studies of sepsis and VAP, and to evaluate whether they provide valuable and real information on biological mechanisms. In addition, we want to assess whether they have the potential to reflect "in vitro" the actual pathophysiology of sepsis in VAP.

\section{Pathophysiology of systemic inflammatory response syndrome (SIRS) and VAP}

We will describe as brief as possible, the beginnings and the evolution in the definition of sepsis, SIRS and VAP in order to reach a better understanding of the current definitions.

\section{Sepsis and SIRS}

Sepsis is an important cause of mortality; it constitutes $30 \%$ to $50 \%$ of deaths in ICU patients and this situation amounts to high costs in health (9).

In 1991 sepsis was first defined as a "SIRS to the presence of infection", setting the presence of 2 or more alterations in heart and respiratory rate, body temperature and white blood cell count as criteria; in addition, when sepsis was associated with an organic dysfunction it was called severe sepsis and when it was associated with refractory hypotension, septic shock $(10,11)$.

However, since this definition of SIRS has been implemented, it was seen an excessive use of antibiotics and fluid resuscitation because the criteria were not such specific. That is why in 2016, through the "Sepsis-3 task force" (1-4) the definition of sepsis was updated by the European Society of Medical Care Intensive Care Society and the Critical Care Medicine Society as an infection associated with an excessive immune response of the host with a consequent organ failure.

Thus, complementing the definition, the infection was associated to the increment of the "secondary organ failure assessment" (SOFA) score by 2 or more points from the baseline, and a new set of clinical criteria were approved, called quick SOFA (qSOFA). On the other hand, septic shock is a subset of septic patients, with circulatory and cellular and/or metabolic dysfunction associated with an increased risk of death $(>25 \%)$, which from another perspective is defined as "induced hypotension by sepsis requiring vasopressors and an increased lactate level in the absence of hypovolemia". Following these foundations in the current definitions, we must say that to date there is some concern about its usefulness, since it can delay early identification and intervention in infected patients before they develop organ dysfunction and, moreover, there has been no demonstrated that the use of the new sepsis criteria improves clinical outcomes (12).

\section{VAP}

Patients with VAP, already defined above, should present a new pulmonary infiltrate or progression of a previous one, accompanied by clinical evidence of infection, such as fever, purulent sputum, leukocytosis and/or decreased oxygenation $(13,14)$.

Aspiration of secretions from the oropharynx is the major mechanisms of arrival or the pathogens to the lower respiratory tract. Other mechanisms include contiguity infection, hematogenous, or through the ventilator circuits. Coma or deep sedation, cardio-respiratory arrest, polytrauma and immune suppression are other possible mechanisms.

The process by which ventilated patients develop VAP is of prolonged microaspiration, whereas in the animal models it is due to inoculation of a significant volume of respiratory pathogens to induce the infection.

Additionally, it was proved that MV triggers a sterile inflammatory response in lungs associated with an elevation of levels of cytokines including interleukine (IL)-6, IL$1 \mathrm{a}$ and $-1 \mathrm{~b}$, interferon (IFN) $\gamma$ and tumour necrosis factor (TNF) $\alpha$ and chemokines such as IL-8 $(15,16)$.

Some authors divide VAPs in early and late stages depending on if they are present before the $5^{\text {th }}$ to $7^{\text {th }}$ day of invasive ventilation, or not; this is considered an important fact, since it could be associated with the probability or not of antibiotic resistance. However, others argue that such a definition should be based on hospital admission as a starting point and not at the time or place of intubation, since intubation can take place after several days of hospitalization and thus, the patient is colonized with typically nosocomial pathogens $(14,17)$. 


\section{Looking for the perfect animal model}

The first studies of pneumonia in ventilated animals were published in the 1980s using baboons. Animals, with no lung injury, or lung injury induced by intravenous oleic acid, 0.06 to $0.08 \mathrm{~mL} / \mathrm{kg}$; exposure to $100 \%$ oxygen for 3 to 6 days; or exposure to $80 \%$ oxygen for 6 days, were used. Cultures of tracheal secretions, lung aspirates, bronchoalveolar lavage (BAL) and protected specimen brushes (PSB) were compared with cultures of homogenized material of lung and with histological findings (18).

Most of studies with animals are performed in mammals, more than half of sepsis studies are performed on rodents. Rodent models are frequently used because their small size, reproductive rate, low price and easy to manipulate. However, rodents have many disadvantages for their relevant differences with humans such as anatomy of respiratory tract (they have not respiratory bronchioles, no submucosal glands below trachea, etc.), physiology of respiratory tract (weak cough reflex), pattern recognition receptors (expression and ligand specificity of Toll-like receptors), antimicrobial secretions (pattern of lysozyme secretion) and other important facts for sepsis like lower circulating neutrophils counts, no murine homolog of IL-8 and no alfa-defensins (19). For such reason, large animal species are relatively heterogeneous genetic models that represent in a more similar way the human species than the one provided by rodents (20). Therefore, several large models were used like dogs, cats, pigs, sheep, rabbits and various species of primates in VAP and sepsis research (21). The models of dogs and cats have been mostly replaced by pigs and sheep, due to the public worry for these pet species. Sheep are frequently used in chronic models, particularly in studies investigating pulmonary pathophysiology, because of their submissive nature.

The equivalences between humans and pigs towards to anatomy and renal, cardiovascular, and gastrointestinal physiology make pigs worthy experimental models. Nonhuman primates, including baboons, cynomolgus monkeys and rhesus monkeys are frequently held back for preclinical and small size studies, due to the ethical requirements for the use of the least susceptible species, significant cost and potential for transmission of zoonotic diseases (22).

\section{Preparation of large animal models of VAP}

Marquette et al. investigated in pigs the development of acquired pulmonary infection as a result of long
MV (5). Although a novel model of experimentally produced VAP by oropharyngeal challenge, has been recently described (23), most of the published experience has been done following the Marquette's method original description. This pig model became the standardized model of pig for VAP and since then, it has been used for different purposes (24-28). Clinical studies usually used large white Landrace piglets weighing up to $30 \mathrm{~kg}$ that may make easier the haemodynamic controls during sepsis and replicate in a more reliable way the clinical features of human infections. Before pigs are bronchial inoculated and infected with pathogens, they must receive antibiotics such as ceftriaxone or ampicillin, to prevent infestation and pneumonia caused by endogenous flora. These antibacterial drugs help to protect against common bacteria such as P. multocida, $S$. suis, Klebsiella oxytoca, P. aeruginosa, Staphylococcus epidermidis and $S$. aureus, that colonized the upper airways of pigs. The antibiotics should be administered intravenously, approximately 30 minutes prior to intubation (29). After orotracheal intubation, piglets are ventilated using a volume-controlled ventilator (26). In order to avoid lung atelectasis, both pigs, cows or sheep are ventilated in the prone position and initially ventilated with a tidal volume of $<10 \mathrm{~mL} / \mathrm{kg}$ to prevent iatrogenic ventilator-induced lung injury (30).

\section{Inoculation ways}

Aspiration was studied in $\mathrm{dog}$ and cat models and bacteriologic diagnosis was evaluated in baboons. When animals are haemodynamic stabilized, they can be inoculated, with a fibrobronchoscope, with a high concentrated of bacterial suspension $\left(10^{6}-10^{8} \mathrm{cfu} / \mathrm{mL}\right)$ thus pneumonia is induced by a specific pathogen and the suspension is uniformly distributed throughout the lobes of both lungs (31-33).

Pigs were used for studying either spontaneous or induced VAP. Marquette et al. started a model of experimental pneumonia in mechanically ventilated pigs. The authors found that pigs mechanically ventilated for 4 days, experimentally developed tracheal stenosis consistently started spontaneous pneumonia (34), and hence decided to conduct a study comparing the histologic and bacteriologic findings in previously healthy pigs infected with bacterial pneumonia in contrast with those free of antibiotic therapy (35).

Piglets with prolonged MV and/or experimental bronchial stenosis quickly suffer from endogenous 
pneumonia predominantly due to $P$. multocida or S. suis (36).

Almost all piglets with no antibiotics, but less than half of those receiving ceftriaxone, develop histologically-proven pneumonia $(\mathrm{P}<0.05)$. Pneumonia is present in $54 \%$ of the dependent segments and in only $25 \%$ of the nondependent lung segments $(\mathrm{P}<0.05)$. In $75 \%$ of the cases, pneumonia involves both lungs (37).

\section{VAP and spontaneous lung infection}

Panigada et al., studied long term-ventilation on sheep and the effect of gravity on the aspiration of pathogens of the oropharyngeal after tracheal intubation. They discovered an important role of gravidity in the pathogenesis of VAP and that airway colonisation could be prevented with the horizontal orientation of the tracheal tube in lateral body position maintaining good gas exchange and function of lungs (38). However, pulmonary studies on sheep to human pathology is difficult because of the differences in anatomical conditions. But, the pig model gives a more reliable data of experimental laboratory in the clinical conditions for humans in the ICU (39). However the Panigada's study suggests the important role of the position of the endotracheal tube for preventing aspiration and bacterial contamination of the oropharynx and lower airways in ventilation conditions and furthermore, the pathogenesis of VAP consequence of colonization of endogenous bacterial of the upper airways and gastric pathogens, aspirated through the tracheal tube strike.

\section{Hemodynamic and respiratory parameters}

In 2007 Luna et al. investigated the physiologic and pathological changes of severe pneumonia under MV of 12 piglets inoculated with $P$. aeruginosa, during 72 h, indicating appropriated antibiotic therapy to half of them. They found that tachycardia presented early (HR at time 0 : $129.1 \pm 31.3$ and at 2 hours: $159.8 \pm 18.5 / \mathrm{min}, \mathrm{P}=0.002$ ), diastolic blood pressure fell down at 36 hours and fever reached $39.4{ }^{\circ} \mathrm{C}$ at 24 hours $(\mathrm{P}=0.02)$ compared with basal parameters (Figure 1A). Antibiotic therapy (appropriate or inappropriate) did not altered any clinical or hemodynamic value except for fever, which was remarkably higher in piglets with no antibiotics (Figure 1A).

The most significant finding on blood values was the sharp decreased in the $\mathrm{PaO}_{2} / \mathrm{FiO}_{2}$ ratio, which increasingly worsened up to the final (Figure 1B).

In the course of pneumonia, airway pressures elevated remarkably at 2 hours, while static compliance dropped (Figure 1C). Antibiotic therapy had no effects in lung mechanics (25).

Furthermore, Bruno et al. investigated animal models of VAP evaluating studies of piglets initially ventilated in prone position with a VT of $<10 \mathrm{~mL} / \mathrm{kg}$ to avoid iatrogenic ventilator-induced lung injury. In these studies, they observed a tendency to hypoventilation and respiratory acidosis, therefore a higher $\mathrm{VT}$ per $\mathrm{kg}$ is required in piglets to prevent lung injury because of their bigger lungs in comparison with their weight (29).

\section{Inflammatory response}

When pathogens enter into the alveolar space, they trigger an inflammatory response with the development of defense mechanisms and the production of several mediators and acute phase proteins. This response consists of many proinflammatory cytokines, as IL-1b, IL-6, IL-8, TNF- $\alpha$ to control the progression of infection and to eliminate the pathogens $(34,35)$.

The pro-inflammatory cytokines induce the delivery from the circulatory system of defense cells such as lymphocytes, platelets, and neutrophils to the inflammatory sites (Figures 2 and 3). Later, the infection remains beneficial when it is locally limited by the recruitment of anti-inflammatory cytokines like IL-10 and IL-1 receptor antagonist (grading of pneumonia Table 1). If the inflammatory response is greater and not limited, it can affect negatively in the evolution of the infection.

That is the reason why the measurement of inflammatory mediators detected in serum or BAL, has an important prognostic value and is an important predictor of failure of treatment and mortality in severe pneumonia and $\operatorname{VAP}(36,37)$.

Several studies measured cytokines such as IL-8, IL-6, IL-5, IL-1b and TNF- $\alpha$ in BAL and serum in order to evaluate and compare the local inflammatory response (BAL) with the systemic response (serum) in ventilated piglets with VAP induced by $P$. aeruginosa (26) and with methicillin resistant $S$. aureus (18). The results showed an increased production of pro-inflammatory cytokines in both responses but tended to be higher in BAL in comparison with serum, suggesting the initially compartmentalization of the production of cytokines (26).

Luna et al. (30) studied these inflammatory responses in ventilated piglets with VAP after different antibiotics therapies, showing a lower local and systemic response in 

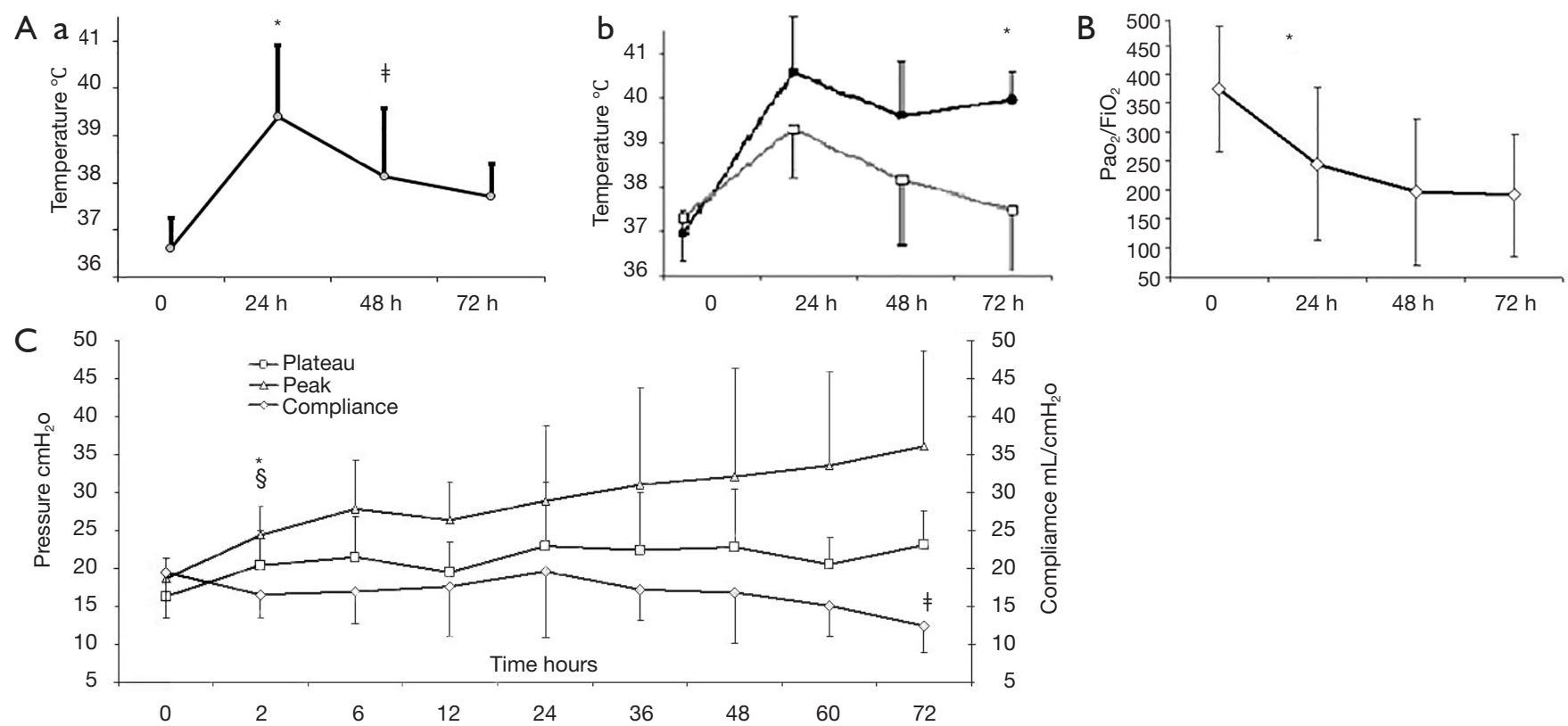

Figure 1 Temperature, $\mathrm{PaO}_{2} / \mathrm{FiO}_{2}$, airway pressures and compliance variations in piglets. (A) (a) After inoculation the temperature rose steeply, being $36.6 \pm 1.0^{\circ} \mathrm{C}$, at baseline and rising to $39.4 \pm 1.4{ }^{\circ} \mathrm{C}$ at 24 hours $\left({ }^{*}\right), \mathrm{P}=0.0002$ and $38.1 \pm 1.8^{\circ} \mathrm{C}$ at 48 hours $\left(^{*}\right)$, $\mathrm{P}=0.021$. (b) body temperature tended to be higher in piglets not receiving ABT, beginning 24 hours after inoculation. This difference became significant at the last measurement before death or sacrifice $\left(^{*}\right) \mathrm{P}=0.018$. (B) $\mathrm{PaO}_{2} / \mathrm{FiO}_{2}$ decreased over time, becoming significant 24 hours after inoculation, basal $=386$ and at 24 hours $=242 \mathrm{mmHg}\left({ }^{*}\right), \mathrm{P}=0.034$. (C) Peak and plateau pressures and compliance in the overall population at the different time points from the time of inoculation to the time of sacrifice. A continuous increase in airway pressures was obvious from the beginning of the experiment achieving significance at 2 hours, $\mathrm{P}=0.003$ for peak $\left(^{*}\right)$ and $\mathrm{P}=0.007$ for plateau (§) pressures. Static compliance reduction became significant at 72 hours $(\ddagger), \mathrm{P}=0.009$. These changes may be attributed to pneumonia and to acute lung injury. According with Luna et al. (25).

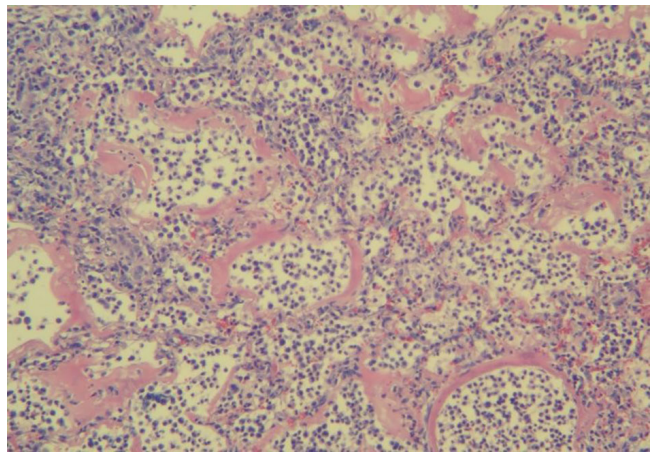

Figure 2 Confluent pneumonia. Micro-photograph showing alveolar spaces with polymorphonuclear leukocytes infiltration. It also shows the alveolar epithelium covered by hyaline membranes. Hematoxylin-eosin 250x. According with Luna et al. (25).

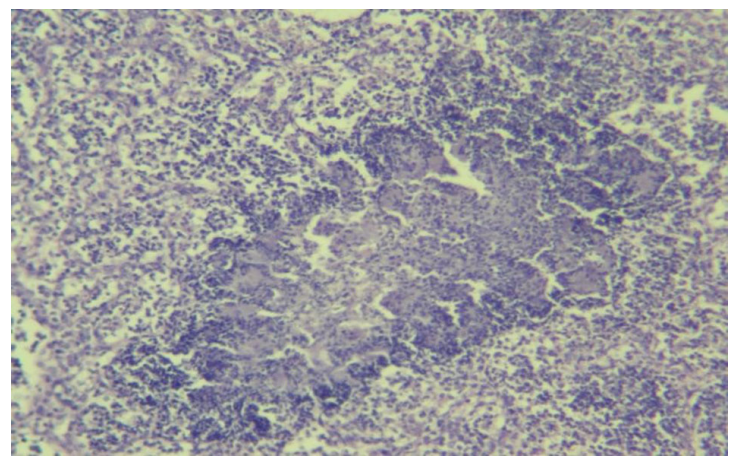

Figure 3 Abscess pneumonia. Consolidation; infiltration of polymorphonuclear leukocytes, fibrinous exudates, and cellular necrosis with disruption of cellular architecture. Hematoxylineosin 250x. According with Luna et al. (25). 
Table 1 Histopathological grading of pneumonia

\begin{tabular}{lll}
\hline Grade & Histologic lesion & Description \\
\hline 0 & No pneumonia & Absence of lesions \\
1 & Mucus plugging & Purulent mucus plugging in peripheral airway \\
2 & Bronchiolitis & $\begin{array}{l}\text { Polymorphonuclear leukocytes localised within the lumen and bronchiolar } \\
\text { wall alterations }\end{array}$ \\
3 & Pneumonia & Focal polymorphonuclear leukocytes, fibrin and few erythrocytes in the \\
& & alveolar lumen \\
5 & Confluent pneumonia & Confluent pneumonia lesions (Figure 2) \\
& Abscessed pneumonia & Pneumonia coexisting with parenchymal necrosis (Figure 3) \\
\hline
\end{tabular}

According with Rouby et al. (40).

piglets after 72 hours of therapy in comparison with those which had not received antibiotics. This result suggests an anti-inflammatory effect in the antibiotic therapy.

\section{Acute lung injury and distress response}

$\mathrm{MV}$ is a significant risk factor for the occurrence of acute lung injury and acute respiratory distress syndrome (41). A frequent evolution of these complications is the multiple organ dysfunction syndrome (42). This syndrome commonly affects kidneys and liver with the elevation of biochemical markers such as creatinine, aspartate aminotransferase (AST) and alanine aminotransferase (ALT). This is an important predictor of mortality, elevating the morbidity and mortality in ventilated patients with and leads to significant morbidity and mortality in these patients, and is a major risk factor for death in patients with distress syndrome (42).

Dhanireddy et al. studied six mice with bacterial ( $S$. aureus or Escherichia coli) aspiration and MV. The aim was to determine whether MV affects the inflammatory response in a mouse model of pulmonary bacterial infection. They studied not only the pro-inflammatory cytokines but also the extra-pulmonary organ dysfunction caused by MV. Serum levels of creatinine, AST and ALT, as measures of kidney and liver injury were increased in the ventilated mice (43). As a mechanism for the development of these injuries it has been suggested the apoptosis (44).

\section{Cardiac events output}

Almost one-third of patients with severe pneumonia by Streptococcus pneumoniae (SP) suffer from adverse cardiac events during or after pneumonia. In several studies was observed that in mice with bacteremia by SP, it can disseminate through the heart, attack the cardiomyocyte, and damage cardiac function. Reyes et al. investigated in nonhuman primate models if SP can invade the myocardium, induce apoptosis of cardiomyocytes, cause cardiac dysfunction and origin cardiac injury during severe pneumonia after antibiotic therapy. They studied severe pneumonia by SP in six non-human primates and three controls animals with no infection. They didn't find specific electrocardiographic and echocardiographic ischemic changes (45).

They measured increased serum levels of heart-type fatty acid binding protein and troponin $\mathrm{T}$ in acute infected animals $(\mathrm{P}<0.05)$. Furthermore $\mathrm{SP}$ was present in the myocardium of the all models with severe pneumonia, detecting the necroptosis and apoptosis of myocardiocytes, followed by cardiac permanent damage (45).

\section{From laboratory animal model to clinical practice}

Although experimental studies in animal models are useful in the study of ventilated severe pulmonary infections, translational medicine has its similitudes and differences with respect to the true reproduction of the mechanisms of VAP in humans.

First at all, VAP is the result of oropharyngeal and upper airways bacterial colonization and subsequent aspiration, but in animal models, pneumonia is due to bronchial inoculation. However, depending on the size of this instillation and the pathogen used, the model may replicate the mechanisms of the majority of VAPs associated with sepsis, but probably not in all of cases. 
In the laboratory, the animals used for models are healthy before they are inoculated and mechanical ventilated. This is not the same scene in UCI, where most of ventilated patients have comorbidities and/or previously antibiotics therapy that influence the evolution and prognosis of VAP. In the other hand, without comorbidities or previous drugs, there is no interference in the appreciation of clinical, inflammatory, microbiological and haemodynamic development of the infection in the ventilated animal models.

\section{Conclusions}

VAP is one of the main causes of nosocomial infection. Animal models of VAP are performed mainly after the inoculation of high concentration of pathogens in the airway and produce a strong dissemination of the infection in the lungs. The results of this challenge are the production of acute lung injury, distress response, cardiac events and immune modulation, resembling very closely severe VAP. This model is reproducible, and thus, it could provide valuable information. Sepsis causes thousands of deaths and expenditures in healthcare worldwide. Novel therapies, beside antibiotics, fluid resuscitation, and basic supportive care, are the main issues of clinical research. However, the search for new targets to improve care, to provide platforms for testing different hypotheses and to provide newer approaches to manage this complex disease, continues, and for doing this, animal models are necessary.

\section{Acknowledgements}

None.

\section{Footnote}

Conflicts of Interest: The authors have no conflicts of interest to declare.

\section{References}

1. American Thoracic Society; Infectious Diseases Society of America. Guidelines for the Management of Adults with Hospital-acquired, Ventilator-associated, and Healthcareassociated Pneumonia. Am J Respir Crit Care Med 2005;171:388-416.

2. Torres A, Niederman MS, Chastre J, et al. International ERS/ESICM/ESCMID/ALAT guidelines for the management of hospital-acquired pneumonia and ventilator-associated pneumonia: Guidelines for the management of hospital-acquired pneumonia (HAP)/ ventilator-associated pneumonia (VAP) of the European Respiratory Society (ERS), European Society of Intensive Care Medicine (ESICM), European Society of Clinical Microbiology and Infectious Diseases (ESCMID) and Asociación Latinoamericana del Tórax (ALAT). Eur Respir J 2017;50. pii: 1700582.

3. Singer M, Deutschman CS, Seymour CW, et al. The Third International Consensus Definitions for Sepsis and Septic Shock (Sepsis-3). JAMA 2016;315:801-10.

4. Wichterman KA, Baue AE, Chaudry IH. Sepsis and septic shock: a review of laboratory models and a proposal. J Surg Res 1980;29:189-201.

5. Marquette $\mathrm{CH}$, Wermert D, Wallet F, et al. Characterization of an animal model of ventilator-acquired pneumonia. Chest 1999;115:200-9.

6. Prince AS, Mizgerd JP, Wiener-Kronish J, et al. Cell signaling underlying the pathophysiology of pneumonia. Am J Physiol Lung Cell Mol Physiol 2006;291:297-300.

7. Shawn JP. Animal models of human pneumonia. Am J Physiol Lung cell Mol Physiol 2008;294:387-98.

8. Fink MP. Animal models of sepsis. Virulence 2014;5:143-53.

9. Liu V, Escobar GJ, Greene JD, et al. Hospital deaths in patients with sepsis from 2 independent cohorts. JAMA 2014;312:90-2.

10. Bone RC, Balk RA, Cerra FB, et al. Definitions for sepsis and organ failure and guidelines for the use of innovative therapies in sepsis. The ACCP/SCCM Consensus Conference Committee. American College of Chest Physicians/Society of Critical Care Medicine. Chest 1992;101:1644-55.

11. Rhodes A, Evans LE, Alhazzani W, et al. Surviving Sepsis Campaign: International Guidelines for Management of Sepsis and Septic Shock: 2016. Intensive Care Med 2017;43:304-77.

12. Gül F, Arslantaş MK, Cinel İ, et al. Changing Definitions of Sepsis. Turk J Anaesthesiol Reanim 2017;45:129-38.

13. Chacko R, Rajan A, Lionel P, et al. Oral decontamination techniques and ventilator-associated pneumonia. Br J Nurs 2017;26:594-9.

14. Kalil AC, Metersky ML, Klompas M, et al. Management of Adults With Hospital-acquired and Ventilatorassociated Pneumonia: 2016 Clinical Practice Guidelines by the Infectious Diseases Society of America and the American Thoracic Society. Clin Infect 
Dis 2016;63:e61-111.

15. Vaneker M, Feico JH, Jan van E, et al. Mechanical ventilation in healthy mice induces reversible pulmonary and systemic cytokine elevation with preserved alveolar integrity: an in vivo model using clinical relevant ventilation settings. Anesthesiology 2007;107:419-26.

16. Ding N, Wang F, Xiao H, et al. Mechanical ventilation enhances HMGB1 expression in an LPS-induced lung injury model. PLoS One 2013;8:e74633.

17. Trouillet JL, Chastre J, Vaugnat A, et al. Ventilatorassociated pneumonia cause by potentially drug-resistant bacteria. Am J Respir Crit Care Med 1998;157:531-9.

18. Johanson WG Jr, Seidenfeld JJ, de los Santos R, et al. Prevention of nosocomial pneumonia using topical and parenteral antimicrobial agents. Am Rev Respir Dis 1988;137:265-72.

19. Baron RM, Choi AJ, Owen CA, et al. Genetically manipulated mouse models of lung disease: potential and pitfalls. Am J Physiol Lung Cell Mol Physiol 2012;302:L485-97.

20. Wagener BM, Pittet JF. A More Clinically Relevant Model of Ventilator-Associated Pneumonia? Anesthesiology 2014;120:1075-7.

21. Deitch EA. Animal models of sepsis and shock: a review and lessons learned. Shock 1998;9:1-11.

22. Fink MP, Heard SO. Laboratory models of sepsis and septic shock. J Surg Res 1990;49:186-96.

23. Li Bassi G, Rigol M, Marti JD, et al. A novel porcine model of ventilator-associated pneumonia caused by oropharyngeal challenge with Pseudomonas aeruginosa. Anesthesiology 2014;120:1205-15.

24. Li Bassi G, Fernandez-Barat L, Saucedo L, et al. Endotracheal tube biofilm translocation in the lateral Trendelenburg position. Crit Care 2015;19:59.

25. Luna CM, Baquero S, Gando S, et al. Experimental Severe Pseudomonas aeruginosa Pneumonia and Antibiotic Therapy in Piglets Receiving Mechanical Ventilation. Chest 2007;132:523-31.

26. Rios Vieira SR, Goldstein I, Lenaour G, et al. Experimental ventilator-associated pneumonia: distribution of lung infection and consequences for lung aeration. Braz J Infect Dis 2003; 7:216-23

27. Sibila O, Luna CM, Agustí C, et al. Effects of glucocorticoids in ventilated piglets with severe pneumonia. Eur Respir J 2008;32:1037-46.

28. Goldstein I, Wallet F, Robert J, et al. Lung tissue concentrations of nebulized amikacin during mechanical ventilation in piglets with healthy lungs. Am J Respir Crit
Care Med 2002;165:171-5.

29. Bruno D, García Morato J, Luna CM. Animal models of VAP. Eur Resp Mon 2011;53:98-112.

30. Luna CM, Bruno DA, García-Morato J, et al. Effect of linezolid compared with glycopeptides in methicillinresistant Staphylococcus aureus severe pneumonia in piglets. Chest 2009;135:1564-71.

31. Goldstein I, Bughalo MT, Marquette CH, et al. Mechanical ventilation-induced air-space enlargement during experimental pneumonia in piglets. Am J Respir Crit Care Med 2001;163:958-64.

32. Goldstein I, Wallet F, Nicolas-Robin A, et al. Lung deposition and efficiency of nebulized amikacin during Escherichia coli pneumonia in ventilated piglets. Am J Respir Crit Care Med 2002;166:1375-81.

33. Sibila O, Martinez-Olondris P, Agusti C, et al. An Experimental Model of Pneumonia Induced by MRSA in Ventilated Piglets. Proc Am Thorac Soc 2007;4:A327.

34. Marquette CH, Mensier E, Copin MC, et al. Experimental models of tracheobronchial stenoses: a useful tool for evaluating airway stents. Ann Thorac Surg 1995;60:651-6.

35. Luna CM, Sibila O, Agusti C, et al. Animal models of ventilator-associated pneumonia. Eur Respir J 2009;33:182-8.

36. Marquette CH, Copin MC, Wallet F, et al. Relationship between microbiologic and histologic features in bacterial pneumonia. Am J Respir Crit Care Med 1996;154:1784-7.

37. Wermert D, Marquette CH, Copin MC, et al. Influence of pulmonary bacteriology and histology on the yield of diagnostic procedures in ventilator-acquired pneumonia. Am J Respir Crit Care Med 1998;158:139-47.

38. Panigada M, Berra I, Greco G, et al. Bacterial colonization of the respiratory tract following tracheal intubation-effect of gravity: an experimental study. Crit Care Med 2003;31 729-37.

39. Stiletto RJ. Bacterial colonization of the respiratory tract under artificial ventilation: is there proof of clinically relevant effects of the endotracheal tube orientation under poistioning therapy? Crit Care Med 2003;31:973-4.

40. Rouby JJ, Martin De Lassale E, Poete P, et al. Nosocomial bronchopneumonia in the critically ill. Histologic and bacteriologic aspects. Am Rev Respir Dis 1992;146:1059-66.

41. Dreyfuss D, Ricard JD. Acute lung injury and bacterial infection. Clin Chest Med 2005;26:105-12.

42. Cocanour CS, Ostrosky-Zeichner L, Peninger M, et al. Cost of a ventilator-associated pneumonia in a shock trauma intensive care unit. Surg Infect (Larchmt) 
2005;6:65-72.

43. Dhanireddy S, Altemeier WA, Matute-Bello G, et al. Mechanical ventilation induces inflammation, lung injury, and extra-pulmonary organ dysfunction in experimental pneumonia. Lab Invest 2006;86:790-9.

44. Wesche DE, Lomas-Neira JL, Perl M, et al. Leukocyte apoptosis and its significance in sepsis and shock. J Leukoc Biol 2005;78:325-37.

45. Reyes LF, Restrepo MI, Hinojosa CA, et al. Severe Pneumococcal Pneumonia Causes Acute Cardiac Toxicity and Subsequent Cardiac Remodeling. Am J Respir Crit Care Med 2017;196:609-20.
Cite this article as: Pulido L, Burgos D, García Morato J, Luna CM. Does animal model on ventilator-associated pneumonia reflect physiopathology of sepsis mechanisms in humans? Ann Transl Med 2017;5(22):452. doi: 10.21037/ atm.2017.11.35 